\title{
Low-Power Hardware Design of Binary Arithmetic Encoder in H.264
}

\author{
Ben Hamida Asma ${ }^{1}$, Nedra Jarray ${ }^{1}$ \\ ${ }^{1}$ Laboratory of Electronic and Micro-Electronic \\ (LAB-IT06) \\ Faculty of Sciences of Monastir \\ 5019, Tunisia
}

\author{
Zitouni Abdelkrim ${ }^{2}$ \\ ${ }^{2}$ College of Education in Jubail, \\ University of Dammam, \\ Saudi Arabia
}

\begin{abstract}
Context-Based Adaptive Binary Arithmetic Coding (CABAC) is a well-known bottleneck in H.264/AVC, owing to the highly serialized calculation and high data dependency of the binary arithmetic encoder. This work presents a hardware architecture for the sub-module binary arithmetic encoder of the CABAC. Moreover, a clock gating technique is inserted into our design for power saving. An FPGA design of the proposed architecture can work at a frequency up to $268 \mathrm{MHz}$ on Virtex 5. The suggested design can achieve $17 \%$ of power consumption saving, which allows it to be applied for low power video coding applications.
\end{abstract}

Keywords-H.264; Binary Arithmetic Encoder (BAE); Contextbased Adaptive Binary Arithmetic Coding (CABAC); clock gating

\section{INTRODUCTION}

In the H.264/AVC standard, two entropy encoders are defined: Context-based Adaptive Variable Length Coding (CAVLC) and Context-based Adaptive Binary Arithmetic Coding (CABAC). The CAVLC is a low-complexity entropy coding technique based on the use of switched contextadaptively sets of variable-length codes. Compared to CABAC, The compression efficiency improvement is obtained at the cost of an inevitable complexity overhead. Software-based complexity analysis results show that switching from CAVLC to CABAC usually leads to complexity increasing by $25-30 \%$ for encoding and $12 \%$ for decoding. As an average, 30-40 cycles are required to encode a single bit on digital signal processors, so it takes thousands of cycles to encode one macroblock, which is unacceptable for real-time video coding applications [2]. Therefore, a hardware implementation of $\mathrm{CABAC}$ encoder is always required. However, the bit-serial nature of the CABAC algorithm and the strong data dependency between contiguous bits make it hard to improve the throughput and to parallelize the encoding process.

Hence, a lot of work has been proposed to improve the throughput of the CABAC by processing more than one bin in a single cycle. Yuan Li et al. put forward in [3] a highthroughput low-latency arithmetic encoder (AE) design suitable for HD real-time applications, utilizing a macroblock level pipeline. This design could achieve a throughput of $2 \sim 4$ bins per cycle sufficient for real-time encoding. In [4], a software-hardware codesign for a whole entropy coder was suggested, which took Binary Arithmetic Encoder (BAE) module for the H.264/AVC CABAC entropy encoder as a hardware accelerator. Vagner Rosa et al. presented in [5] a hardware proposal of BAE. The throughput was improved by developing three different architectures of the renormalization step, presenting a processing rate from 0.68 to 1 bin per clock cycle. An RDO-support CABAC encoder was given by [6] and [7] to achieve the bit-rate saving of around 20 percent. In [6], an FPGA-based RISC CPU extension was proposed to accelerate the CABAC in a rate-distortion framework. This design achieved a coding speed of 1 bin per cycle and a clock frequency of $100 \mathrm{MHz}$. In [7], an efficient memory access was suggested to reduce the access frequency of the context RAM.

Most studies have mainly focused on ameliorating the throughput, but limited attention has been paid to reduce power consumption. Therefore, this paper aims to design BAE including a low-power technique. The main contributions of this paper are outlined as follows:

1) We implement a hardware of the BAE, which is the bottleneck of CABAC.

2) We further insert a low-power technique into the BAE architecture. In fact, a clock-gating technique is added into the design of a BAE sub-module, achieving reduced power consumption at a minor implementation effort.

The rest of this paper is organized as follows. Section II presents the CABAC encoding algorithm. Section III shows both encoding processes of the binary arithmetic coder and their corresponding proposed architecture .Section IV provides the FPGA synthesis results, and section V concludes the paper.

\section{CABAC ENCODING ALGORITHM IN H.264}

As presented in Fig. 1, CABAC encoding consists of three main functions: binarization, context modeling, and binary arithmetic coding. The binarization part permits mapping the non-binary valued syntax elements into binary symbols, also known as bins or a bin string. Then each bin is arithmetically coded by a regular coding engine or a bypass coding engine. In the regular coding engine, a context model is used to encode each bin. In the bypass encoding engine, the context is not needed due to the equivalent probability of the appearance of these bins. 


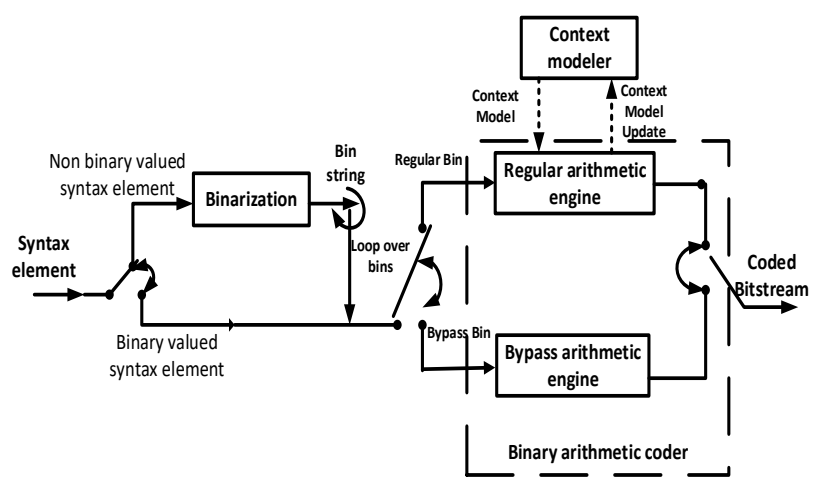

Fig. 1. Diagram of the CABAC encoder.

\section{A. Binarization}

In the binarization process, each syntax element is converted into a bin string. This step is done with different schemes: unary, truncated unary, fixed length and parameterized exp-Golomb. Each task is dedicated to some types of syntax elements, as given in Table 1 . The input and output of the binarization process are the mapped syntax elements and the Context Index (CtxIdx) information. The next step is to use the CtxIdx information to fetch the context model from the context table.

TABLE. I. SyntAX ELEMENTS AND AsSOCIATED TyPES OF BINARIZATION [1]

\begin{tabular}{|l|l|}
\hline Syntax element & Binarization Method \\
\hline mb_type & Table mapping \\
\hline mb_skip_flag & Fixed length \\
\hline Sub_mb & Table mapping \\
\hline Ref_indx_10 & Unary \\
\hline Ref_indx_11 & Unary \\
\hline mvd_10 & $\begin{array}{l}\text { Truncated unary and } \text { exp-Golomb } \\
\text { with }=3, \text { truncated value9 }\end{array}$ \\
\hline mvd_11 & $\begin{array}{l}\text { Truncated } \text { unary and } \text { exp-Golomb } \\
\text { with }=3, \text { truncated value9 }\end{array}$ \\
\hline Intra4x4_pre_mode & Fixed_length \\
\hline rem_intra_4x4_pre-mode & Fixed_length \\
\hline Chroma_pre_mode & Fixed_length \\
\hline Coded_block_pattern & $\begin{array}{l}\text { Fixed length and truncated } \\
\text { unary,truncated value 2 }\end{array}$ \\
\hline Mb_qp_delta & Unary and table mapiing \\
\hline Coded_block_flag & Fixed_length \\
\hline Significant_coefficient_flag & Fixed length \\
\hline Last_significant_flag & Fixed length \\
\hline Coeff_abs_level_minus1 & $\begin{array}{l}\text { Truncated unary, exp-Golomb with } \\
=3 \text { and truncated value 14 }\end{array}$ \\
\hline Coeff_sig_flag & Fixed length \\
\hline End_slice_length & Fixed_length \\
\hline
\end{tabular}

\section{B. Context modeling}

A context model is a probabilistic model with a statistical occurrence rate for each symbol, such that each type of syntax elements has a set of 399 context models as defined by the H.264 standard documentation [1]. Each context model comprises 6-bits representing the Probability State Indices (pStateIdx) and a 7th bit representing the value of the Most Probable Symbol (MPS).

\section{Arithmetic coding}

The aim of the arithmetic encoding process is to generate a bit stream from reading the bins and their context models, if the latter exist. Its principle is based on the division of an initial interval into two sub-intervals according to the context model (Fig. 2). One of two sub-intervals corresponds to the MPS, and the other refers to the Less Probable Symbol (LPS). After that, one of the two intervals is selected asa new one according to the bin value (MPS or LPS). Each interval is defined by two values: range (the length of the interval) and low (the bottom of the interval). These rules determine the updated value of the interval as follows:

$$
\begin{aligned}
\text { If bin }= & L P S \\
& \text { New range }=r L P S(\text { range of } L P S) \\
& \text { New low }=\text { low } \\
\text { If bin }= & M P S \\
& \text { New range }=\text { range }-r L P S=r M P S \text { (range of MPS) } \\
& \text { New low }=\text { low }+ \text { rLPS }
\end{aligned}
$$

Where, the value of rLPS is indexed by pStateIdx, read from context modeling.

For the bins that have the same probability, no context model is needed; and the bins are coded by a simpler bypass coding engine within a CABAC module.
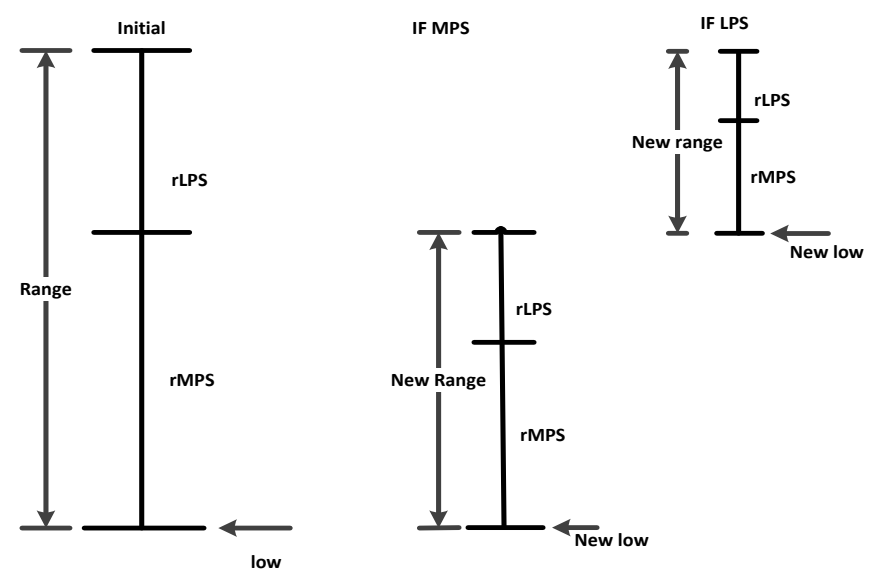

Fig. 2. Interval subdivision process of CABAC.

\section{PROPOSED HARDWARE ARCHITECTURE OF BINARY ARITHMETIC CODER}

At the binarization process, the syntax element of each MB can be treated in parallel. However, at the binary arithmetic coding process, all bin strings should be encoded sequentially. Thus, the binary arithmetic coder is the critical block that affects the throughput. This section firstly presents the processing flow of both regular and bypass BAE modes, and then its provides their corresponding hardware architectures. The clock gating technique is also presented in this section.

\section{A. Regular BAE process and its proposed architecture}

\section{1) Regular BAE process}

The regular BAE process is illustrated in Fig. 3. The chart consists of three steps: interval subdivision, probability-model updating and regular renormalization. 
In the interval subdivision, the interval value is updated according to whether the current input bin (binval) is an MPS or not. The probability model pStateIdx is updated through two tables: TransIdxLPS and TransIdxMPS. The TransIdxMPS is selected when the bin value is equal to an MPS. Otherwise, the TransIdxLPS is used. The final update for low and range values is done by the regular renormalization process, which is needed to keep the interval range between 256 and 512. Fig. 4 shows the flowchart of the regular renormalization.

\section{2) Regular arithmetic coder architecture}

The hardware design of a regular BAE is depected in Fig. 5. It consists of three main modules: probability-model updating, interval subdivision module, and regular renormalization.

The module of probability-model updating is constituted by three principal steps: context model read, context model update, and context read. When the context is read out, the context model will be updated according to bin value through the ROM of either TransIdxLPS or TransIdxMPS. Next, the new context model will be written back to the context table.

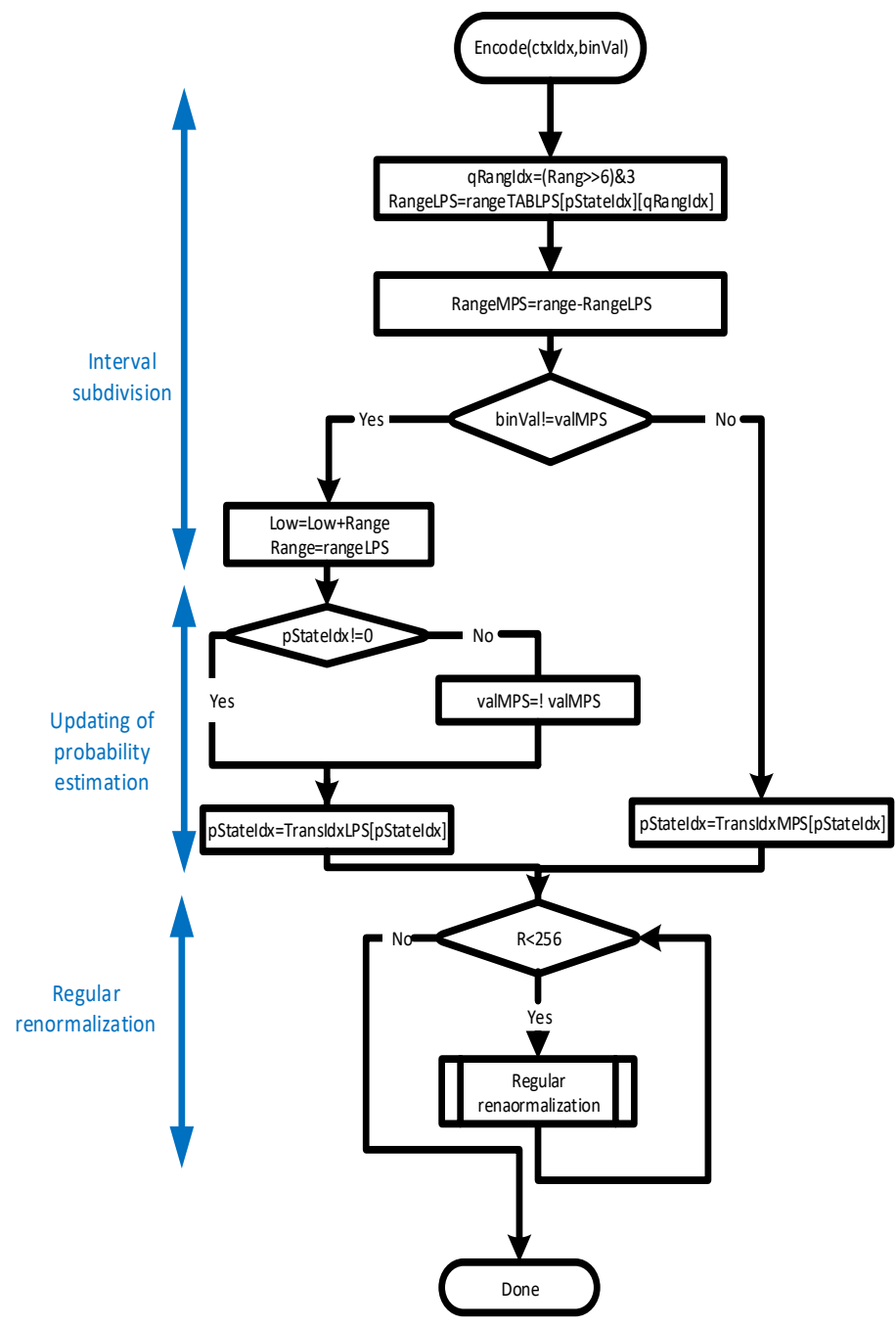

Fig. 3. Regular arithmetic encoding flowchart (from [1] with some modifications)

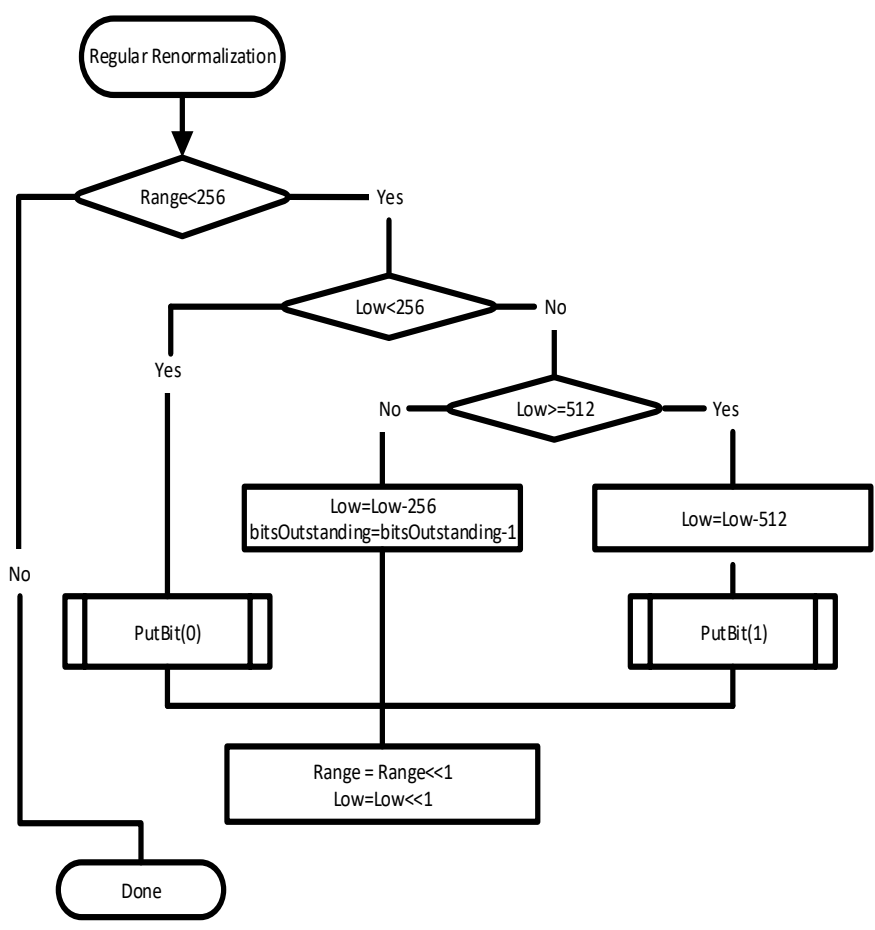

Fig. 4. Regular renormalization flowchart [1].

The module of interval subdivision will be performed when the context model pStateId $x$ is read from the RAM of the context table. Both pStateIdx [5:0] and range [7:6] are used to index the rLPS value from the rLPStable. After that, the interval values (range and low) are calculated by using a ten-bit adder and ten-bit subtractor. According to the bin value, the two top and low multiplexers will select the appropriate value of low and range, respectively.

The module of regular renormalization will be carried after encoding each bin, when the range value is decreased to less than 256. This module is implemented by a finite state machine.

\section{Bypass BAE PROCESS AND ITS PROPOSED ARCHITECTURE}

\section{A. Bypass BAE process}

For the bypass mode, the bin is coded using a coding decision process. The context modeling is skipped as the bins show almost an equiprobable behavior. This encoding mode is a much simpler encoding process compared to the regular mode. Fig. 6 illustrates the bypass process, including the interval subdivision stage and the renormalization stage. There is no iteration loop in the renormalization process in the bypass mode unlike the renormalization in the regular coding mode.

\section{B. Bypass BAE architecture}

A hardware design of the bypass BAE is depicted in Fig. 7. Bypass coding generates valid coding states that conform to equations shown in the flowchart of Fig. 6. This mode is faster than the regular mode since there is no context modeling process. In addition, it is to note that the there is no loop presented in bypass renormalization module. This latter is implemented by a simple finite state machine. 


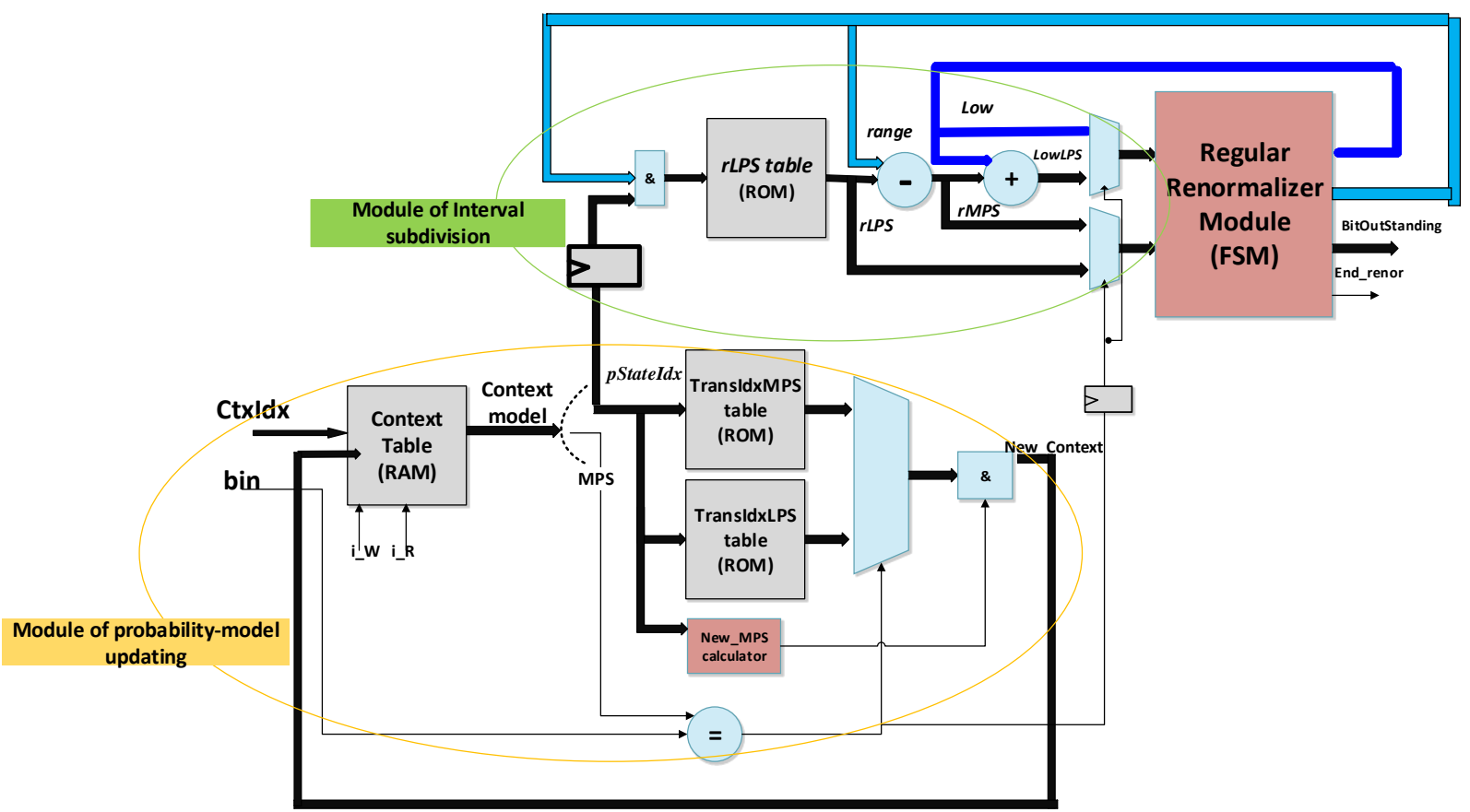

Fig. 5. Architecture representation of regular BAE.

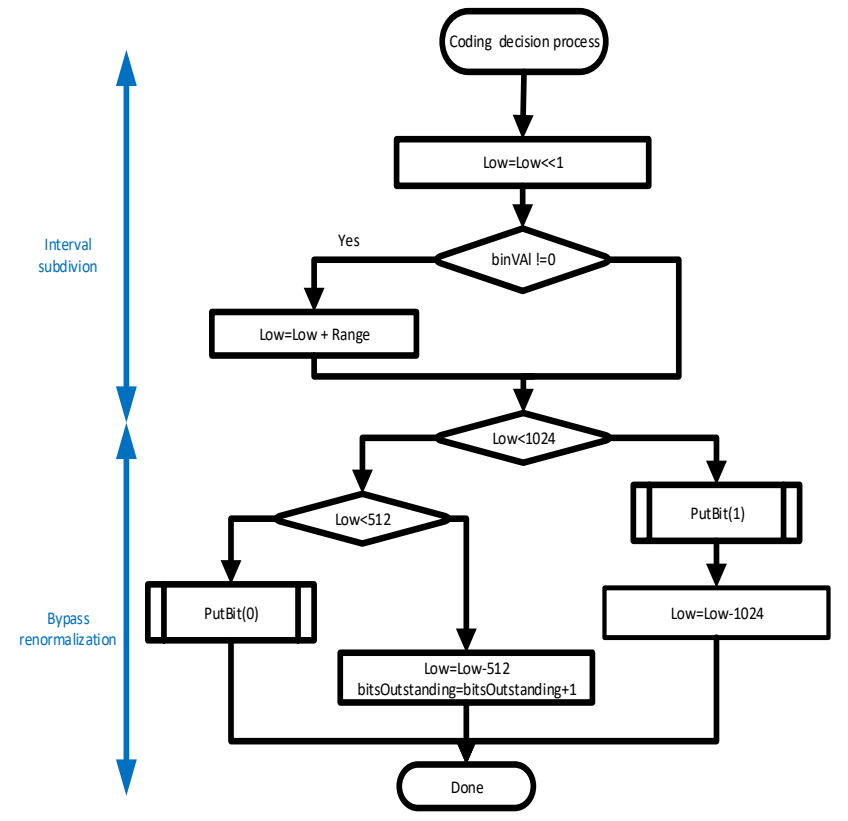

Fig. 6. Bypass BAE flowchart [1].

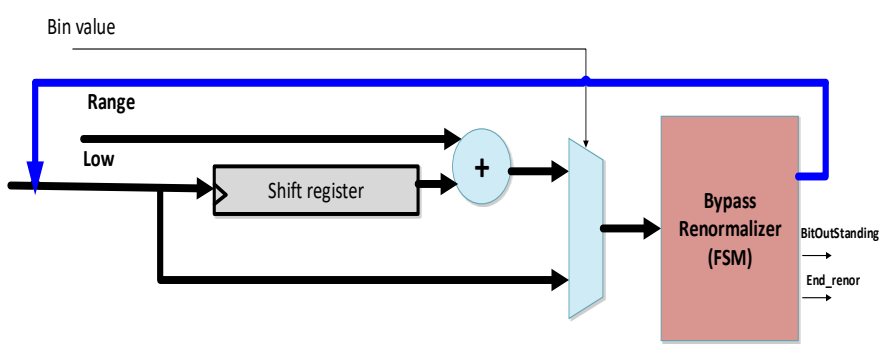

Fig. 7. Architecture representation of bypass BAE.

\section{Clock-Gating TeChNiQue}

Clock gating is among the techniques that are used for reducing dynamic power dissipation. This technique saves power by taking the enable conditions attached to registers and uses them to gate the clock.

At each input bin coming from the binarizer, one of the two coding modes (regular or bypass) is selected. The clock gating technique is inserted to prune the clock either for a regular arithmetic engine or for a bypass coder (i.e. by disabling the flip-flop registers in them).

The practical approach to insert the clock-gating technique in our proposed arithmetic coder is shown in Fig. 8. To avoid the glitch problem caused by clock switching, we use a latchbased clock-gating style.

\section{IMPLEMENTATION RESULTS}

Our design is synthesized and simulated by using the XILINX ISE and ModelSim tools, respectively. The synthesized circuit area of each component of the encoder is listed in Table 2. Synthesis results demonstrate that the BAE can work properly at a clock frequency of $268.5 \mathrm{MHz}$.

The design occupies 300 slices of which a regular BAE unit occupied $82 \%$. It is to clear that the bypass BAE operates at a higher clock frequency compared to the regular mode.

Table 3 presents a comparison with previous work. Our design uses a higher frequency compared to the work [5], which was implemented in the same FPGA technology. Moreover, it is evident that the proposed architecture will achieve the lowest power consumption relative to power consumption of [7] when it is designed on ASIC-based technology. Indeed, as explained in [9], [10] and [11], the power consumption of ASIC designs was observed as being between 3 to 10 times greater than FPGA designs. 


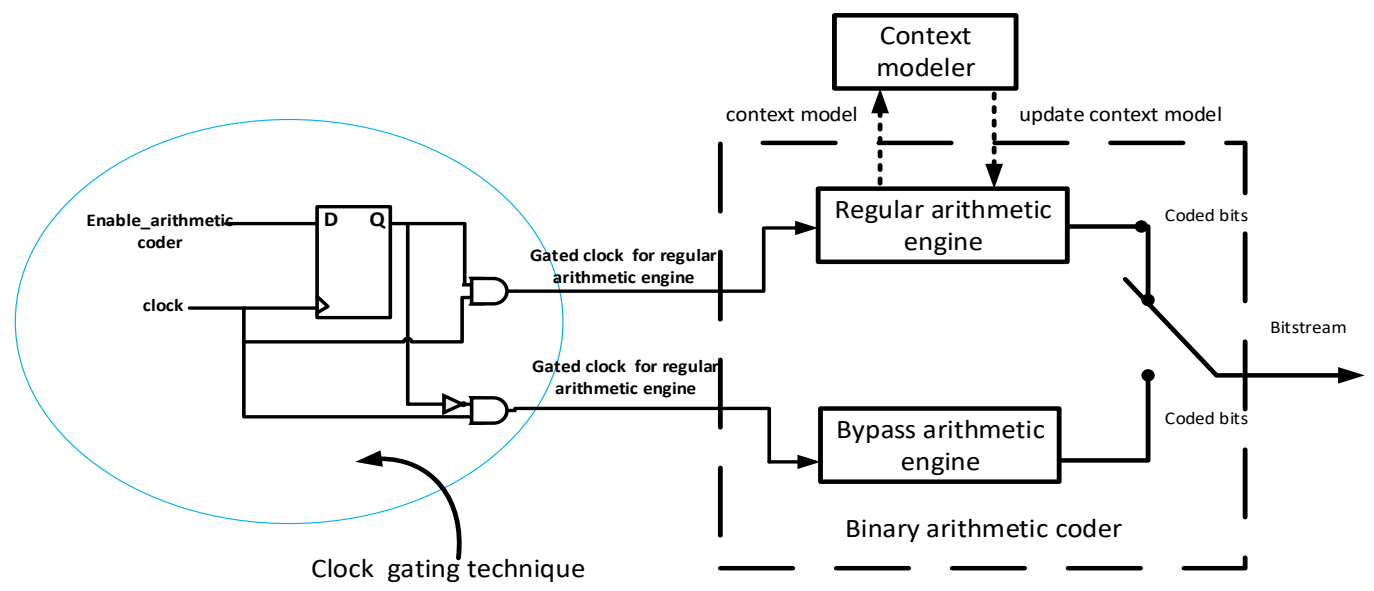

Fig. 8. Diagram of BAE with clock-gating technique.

TABLE. II. SyNTHESIS RESULTS OF EACH BAE UNITS ON VIRTEX 5

\begin{tabular}{|l|l|l|}
\hline Unit Name & $\begin{array}{l}\text { Area } \\
\text { (slices) }\end{array}$ & $\begin{array}{l}\text { Frequency } \\
\text { (MHz) }\end{array}$ \\
\hline Regular BAE & 247 & 268.516 \\
\hline Bypass BAE & 51 & 417.81 \\
\hline Total BAE (without clock gating) & 298 & 268.516 \\
\hline Total BAE(with clock gating) & 300 & 268.516 \\
\hline
\end{tabular}

TABLE. III. COMPARISON OF PERFORMANCE RESUlts

\begin{tabular}{|c|c|c|c|c|c|}
\hline & $\begin{array}{l}\text { Process } \\
\text { technology }\end{array}$ & $\begin{array}{l}\text { Clock } \\
\text { frequency } \\
(\mathrm{MHz})\end{array}$ & $\begin{array}{l}\text { Circuit } \\
\text { Area } \\
\text { (LUT } \\
\text { slices) }\end{array}$ & $\begin{array}{l}\text { Total } \\
\text { power } \\
(\mathrm{mW})\end{array}$ & $\begin{array}{l}\text { Design } \\
\text { parts }\end{array}$ \\
\hline [5] & Virtex 5 & 189 & 436 & $\mathrm{Na}$ & BAE \\
\hline [6] & Startix II & 130 & 603 & $\mathrm{Na}$ & $\begin{array}{l}\text { Total } \\
\text { CABAC }\end{array}$ \\
\hline \multirow{2}{*}{ [7] } & Virtex4 FPGA & 145 & 2559 & $\mathrm{Na}$ & \multirow{2}{*}{$\begin{array}{l}\text { Total } \\
\text { CABAC }\end{array}$} \\
\hline & ASIC $0.13 \mu \mathrm{m}$ & 200 & $\mathrm{Na}$ & 26.6 & \\
\hline$[8]$ & ASIC $0.15 \mu \mathrm{m}$ & 333 & $\begin{array}{l}13.3 \mathrm{~K} \\
\text { gates }\end{array}$ & $\mathrm{Na}$ & $\begin{array}{l}\text { Total } \\
\text { CABAC }\end{array}$ \\
\hline \multirow{2}{*}{ Proposed } & Virtex4 FPGA & 219.479 & 298 & 43 & \multirow{2}{*}{ BAE } \\
\hline & Virtex5 FPGA & 268.516 & 300 & 17.77 & \\
\hline
\end{tabular}

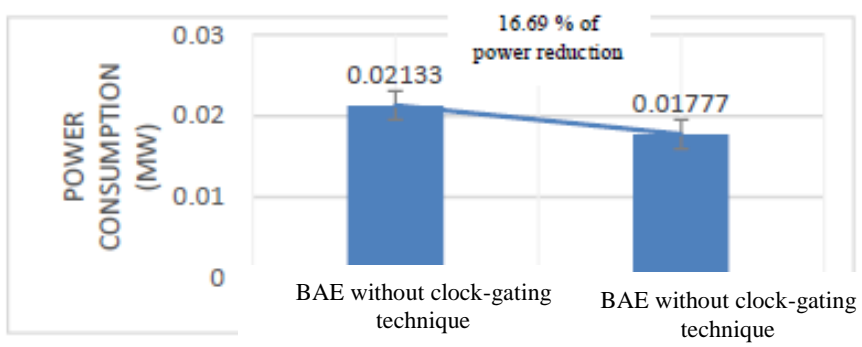

Fig. 9. Diagram of dynamic power consumption of our proposed BAE

Fig. 9 shows the power consumption for both designs (BAE without clock gating and BAE with clock gating). With the insertion of a clock-gating technique, there is about $17 \%$ of dynamic power consumption reduction.

\section{CONCLUSION}

In this paper, our design has focused on the BAE that presents the critical sub-block of the CABAC. Furthermore, a clock-gating technique has been employed to reduce the power consumption. As a result, power consumption can be reduced by about $17 \%$. Therefore, our design can be suitable for low power video coding applications. The synthesis results on Virtex 5 have indicated that the design is capable of operating at $268.516 \mathrm{MHz}$. Finally, it is important to mention that our BAE can fit both H.264/AVC and HEVC formats.

\section{REFERENCES}

[1] Joint Video Team (JVT) of ITU-T VCEG and ISO/IEC MPEG, Draft ITU-T Recommendation and final draft in- ternational standard of joint video specification ITU-T Rec. H.264/ISO/IEC 14496-10 AVC, May 2003.

[2] Yu, Wei, and Yun He. "A high performance CABAC decoding architecture." IEEE Transactions on Consumer Electronics 51.4 (2005): 1352-1359.

[3] Y. Li, S. Zhang, H. Jia, X. Xie, and W. Gao, "A high-throughput lowlatency arithmetic encoder design for HDTV," in IEEE International Symposium on Circuits and Systems, 2013, pp. 998-1001.

[4] R. R. Osorio and J. D. Bruguera,"A New Architecture for Fast Arithmetic Coding in H.264 Advanced Video Coder", 8th Euromicro Conference on Digital System Design (DSD'05)

[5] V. Rosa, L. Max, S. Bampi," High Performance Architectures for the Arithmetic Encoder of the H.264/AVC CABAC Entropy Coder", Electronics, Circuits, and Systems (ICECS), 2010 17th IEEE International Conference on

[6] Y.L. Nunez-Yanez, V.A. Chouliaras, et al., "Hardware-assisted rate distortion optimization with embedded CABAC accelerator for the H.264 advanced video codec", IEEE Trans. CE, vol. 52, no. 2, pp. 590 597, May 2006.

[7] X.H. Tian, T.M. Le SM-IEEE, X. Jiang, Y. Lian SM-IEEE," A HW CABAC Encoder with Efficient Context Access Scheme for H.264/AVC", 2008 IEEE International Symposium on Circuits and Systems

[8] C. Jian-long, L. Yu-kun and C. Tian-sheuan, member, iee,,"a low cost context adaptive arithmetic coder for h.264/mpeg-4 avc video coding",ieee international conference on acoustics, speech and signal processing, 2007. icassp 2007

[9] A. Amara, F. Amiel, T. Ea, "FPGA vs. ASIC for low power applications", Microelectronics Journal, Vol. 37, p. 669-677, January 2006.

[10] A. Chang and W. J. Dally. Explaining the gap between ASIC and custom power: a custom perspective. In DAC '05, pages $281\{284$, new york, ny, usa, 2005. acm press.

[11] D. G. Chinnery and K. Keutzer. Closing the power gap between ASIC and custom: an ASIC perspective. In DAC '05, pages 275, 280. 